\title{
A reflected wave superposition method for vibration and energy of a travelling string
}

\author{
E.W. Chen ${ }^{\mathrm{a}, *}$, Q. Luo ${ }^{\mathrm{a}}$, N.S. Ferguson ${ }^{\mathrm{b}}$, Y.M. Lu ${ }^{\mathrm{a}}$ \\ ${ }^{a}$ School of Mechanical Engineering, Hefei University of Technology, Hefei, 230009, China \\ ${ }^{\mathrm{b}}$ Institute of Sound and Vibration Research, University of Southampton, Southampton SO17 \\ 1BJ, England, UK
}

\begin{abstract}
This paper considers the analytical free time domain response and energy in an axially translating and laterally vibrating string. The domain of the string is either a constant or variable length, dependent upon the general initial conditions. The translating tensioned strings possess either fixed-fixed or fixed-free boundaries. A reflected wave superposition method is presented as an alternative analytical solution for a finite translating string. Firstly, the cycles of vibration for both constant and variable length strings are provided, which for the latter are dependent upon the variable string length. Each cycle is divided into three time intervals according to the magnitude and the direction of the translating string velocity. Applying d'Alembert's method combined with the reflection properties, expressions for the reflected waves at the two boundaries are obtained. Subsequently, superposition of all of the incident and reflected waves provides results for the free vibration of the string over the three time intervals. The variation in the total mechanical energy of the string system is also shown. The accuracy and efficiency of the proposed method are confirmed numerically by comparison to simulations produced using a Newmark-Beta method solution and an existing state space function representation of the string dynamics.
\end{abstract}

Keywords: travelling string, boundary condition, propagating wave, finite length, d'Alembert method, mechanical energy

\section{Introduction}

In the present paper, the lateral vibration of a uniform finite length string modelled with uniform density $\rho$, which travels over two smooth supports under constant speed $v$ and under a constant tension $T$, is considered using the concept of wave propagation. This model can be used to study the lateral vibration of many 
manufacturing technologies and devices involving axially travelling materials, such as conveyor belts, elevator cables, power transmission belts and magnetic tapes. Wave propagation and reflection phenomena in one-dimensional wave bearing systems, such as strings, have been studied for many years; it is though still of great research interest due to their theoretical importance and application. For instance, the classical d'Alembert principle was used to study the reflection phenomenon in either an infinite or a semi-infinite stationary string with classical boundary conditions[1,2]. Recently, Akkaya, Gaiko and Van Horssen[3,4] applied the same method to obtain the exact free, linear, lateral vibration of both a stationary[3] and an axially travelling[4] semi-infinite string. Various alternative approaches have been applied to solve and obtain the response of axially moving materials. Yang and Tan[5] studied both a travelling string and beam using a transfer function method, which for the latter considered a damped, axially moving beam over a set of different boundary conditions. Based on the transfer function formulation and wave propagation, Tan and Ying[6] subsequently derived an exact solution for the response of a translating string with general boundary conditions. Van Horssen[7] used a Laplace transform method instead, constructing exact solutions of the lateral vibrations in travelling strings due to small lateral vibrations of the supports.

Lee[8] analyzed free vibration of a string with time-varying length, by dealing with travelling waves and obtained an exact solution. Simple models which describe these vibrations can be expressed as initial-boundary value problems. Darmawijoyo[9,10] studied such an initial-boundary value problem with a non-classical boundary condition, constructing asymptotic approximations of the solution for an axially travelling string by a multiple-timescales perturbation method. Chen and Ferguson[11] more recently studied the lateral vibration and the energy dissipation of a travelling string attached to a viscous damper at one end, using a time varying state space function method and the Newmark-Beta method. In terms of dissipative boundaries, Gaiko and Van Horssen[12] also gave a complete and accurate description of the damping and the low frequency oscillatory behaviour of the travelling string with an attached spring-mass-dashpot system at one end.

In contrast to previous work on a stationary string and a travelling string defined on an infinite or a semi-finite domain, which either has one or no wave reflections, the present work focuses on the analytical free lateral vibration of an axially translating string with either a constant or varying length. The main difference, as well as the difficulty, lies in the multiple reflections that will exist in a finite domain, which makes the problem more complicated than previous work. A reflected wave superposition method is proposed and completely developed in this study. At both ends of the axially travelling string the multiple reflections of the propagating waves 
are studied. The combined total superposition of the incident and reflected waves constitute the resulting free vibration. This work provides an analytical methodology to solve the translating media problem defined over a finite domain with different boundary conditions and the details of the process are given. This paper is organized as follows. Section 2 introduces the equation of motion describing the lateral vibration of an axially travelling finite string and the relevant boundary conditions. In Section 3, the reflected wave superposition method is used to obtain the response due to the initial conditions of a travelling string between two types of boundaries separated by a constant distance, i.e. the length of string between the boundaries is also constant. Next, Section 4 investigates the time varying cycle and gives an exact analytical response for the time varying length string, i.e. for boundaries that are at either increasing or decreasing distances apart. Section 5 analyzes the total mechanical energy and its time rate of change. Finally, Section 6 provides the main conclusions.

\section{Equations of Vibration}

The equation of motion for the lateral vibration of a travelling string between two boundaries can be obtained by Hamilton Principle $[12,13]$ and is given by

$$
\frac{\partial^{2} w}{\partial t^{2}}+2 v \frac{\partial^{2} w}{\partial t \partial x}+\left(v^{2}-c^{2}\right) \frac{\partial^{2} w}{\partial x^{2}}=0
$$

where $w$ is the lateral displacement, $x$ is the axial coordinate along the length of the string, $t$ is the time, $v$ is the assumed constant translational speed of the string. $c=\sqrt{T / \rho}$ is the free wave propagation speed, where $T$ is the uniform tension and $\rho$ is the uniform string mass per unit length. The derivation of Eq. (1) is given in Appendix A. The domain for the string $x$ is $0<x<l(t)$ and $l(t)$ is the length of the string. Here, two cases concerning the string length are considered: one is the constant length case, i.e. $l(t)=l_{0}$ and so $v \neq i$; the other is the linearly changing length case, i.e. $l(t)=l_{0}+v t$, and so $v=i$, where $l_{0}$ is the initial length of the string. To avoid the vibrational energy accumulating at one end and to allow any propagating wave in the string direction to be reflected at the other end, the string translational speed $v$ is assumed less than the free wave propagation speed $c$, i.e. $|v|<c$.

Various boundary conditions can exist in real situations, for example, free, fixed, spring-dashpot and mass-spring-dashpot, etc. Although only classical boundary conditions are analyzed in this paper, i.e. the free end and the fixed end, the proposed method can also be applied to the nonclassical boundary conditions.

The general one-dimensional wave solution of Eq. (1) for the string displacement using the d'Alembert method is well known [2,14]. It is given by

$$
w(x, t)=F\left(x-v_{r} t\right)+G\left(x+v_{l} t\right)
$$

Here, $v_{l}=c-v$ is the wave propagation speed according to the fixed coordinate 
system as a wave travels from right to left and $v_{r}=c+v$ is the wave speed in the opposite direction. $F\left(x-v_{r} t\right)$ is the right-propagating wave with speed of $v_{r}$ and $G\left(x+v_{l} t\right)$ is the left-propagating wave with speed of $v_{l}$. In Appendix B, it is shown that the d'Alembert method can also be used in the case of Eq. (1). The initial vibration conditions for the string displacement and velocity are assumed as follows:

$$
\left\{\begin{array}{l}
w(x, 0)=\varphi(x) \\
w_{t}(x, 0)=\psi(x)
\end{array},(0 \leq x \leq l(t))\right.
$$

where $w_{t}$ is the first order partial derivation of $w(x, t)$ with respect to time $t$. Then, the initial conditions are satisfied if one has

$$
\begin{aligned}
& F(x)=\frac{1}{2 c} v_{l} \varphi(x)+\frac{1}{2 c} \int_{x}^{l(t)} \psi(\xi) \mathrm{d} \xi+K \\
& G(x)=\frac{1}{2 c} v_{r} \varphi(x)-\frac{1}{2 c} \int_{x}^{l(t)} \psi(\xi) \mathrm{d} \xi-K
\end{aligned}
$$

where $K$ is a constant of integration.

From Eq. (2), the general solution comprises two propagating waves travelling in opposite directions due to the initial condition shown in Fig.1.

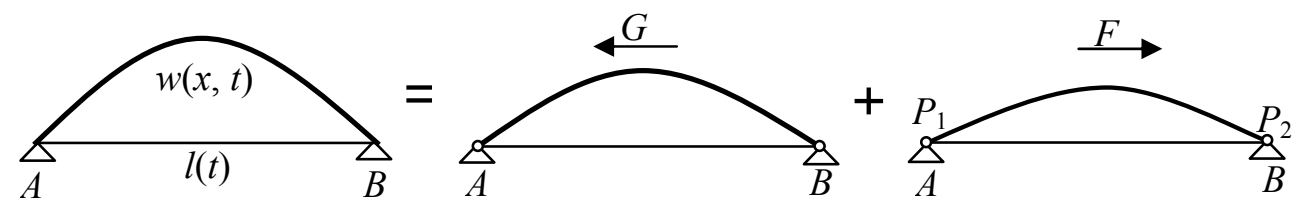

Fig. 1. Initial displacement composed by two propagating waves

Here, $F$ is defined as the wave propagating from left to right and $G$ the wave propagating from right to left. The two propagating waves are reflected at the two boundaries back and forth. So, the total response of the string at any point is the combination of the incident waves and the reflected waves for both the $F$ and $G$ waves. It is obvious that the initial general solution Eq. (2) is not complete, because it does not include the reflected waves. Next, the detailed expressions for the incident and reflected waves for both the $F$ and $G$ waves are given.

\section{A travelling string with constant length}

In the constant length string case, i.e. $l(t)=l_{0}$, the waves $F$ and $G$ will return to their initial states after two reflections at and travelling between the two opposite boundaries. The minimum cycle or time $T_{0}$ required to return to the initial state for these waves $F$ and $G$ is given by [15]

$$
T_{0}=\frac{l_{0}}{v_{l}}+\frac{l_{0}}{v_{r}}=\frac{2 c l_{0}}{v_{l} v_{r}}
$$

where $v_{l}$ and $v_{r}$ are the wave propagation speeds to the left and to the right, 
respectively, according to the fixed coordinate system. It is convenient to define $t_{r}$ as the time required for the point $P_{1}$ on the left end of $F$ to move from end $A$ to $B$ and $t_{l}$ as the time required for the point $P_{2}$ on the right end of $F$ to move from $B$ to $A$, so one has $t_{r}=l_{0} / v_{r}$ and $t_{l}=l_{0} / v_{l}$. If $v>0$, one can define $t_{a}=t_{r}$ and $t_{b}=t_{l}$, or if $v<0$, i.e. the string travelling in the opposite direction, so define $t_{a}=t_{l}$ and $t_{b}=t_{r}$, so that one can get $0<t_{a}<t_{b}<T_{0}$. Based on this, $T_{0}$ is divided into three time intervals: [0, $\left.t_{a}\right]$, $\left[t_{a}, t_{b}\right]$ and $\left[t_{b}, T_{0}\right]$. The detailed expressions for the propagating waves are given next for these three time intervals for the boundaries being either the fixed-fixed or fixed-free case.

\subsection{Fixed-fixed case}

The fixed supports of the string at $x=0$ and $x=l_{0}$ are represented by the boundary conditions:

$$
\left\{\begin{array}{l}
w(0, t)=0 \\
w\left(l_{0}, t\right)=0
\end{array}\right.
$$

3.1.1. The first time interval: $0 \leq t \leq t_{a}$

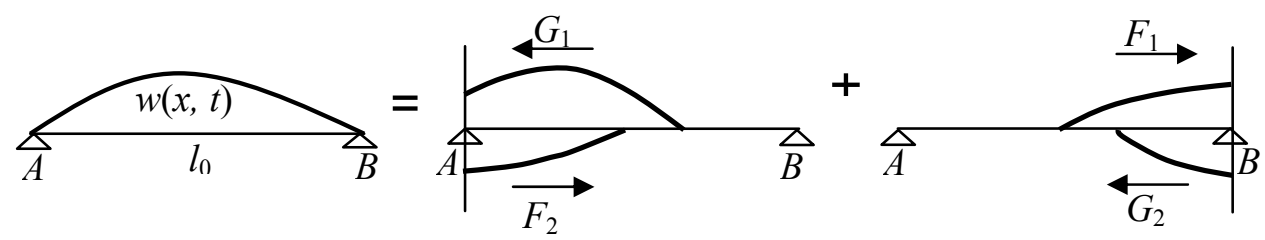

Fig. 2. String deflection comprising the sum of the propagating waves when $0 \leq t \leq t_{a}$

In this time interval, the propagating waves are shown in Fig.2. $G_{2}$ is the reflected wave of $F_{1}$ at $x=l_{0}$ and $F_{2}$ is the reflected wave of $G_{1}$ at $x=0$. According to Eqs. (4) and (5), the expressions for $F_{1}$ and $G_{1}$ are as follows:

$$
\begin{aligned}
& F_{1}\left(x-v_{r} t\right)=\frac{1}{2 c} v_{l} \varphi\left(x-v_{r} t\right)+\frac{1}{2 c} \int_{x-v_{r} t}^{l_{0}} \psi(\xi) \mathrm{d} \xi+K,\left(v_{r} t \leq x \leq l_{0}\right) \\
& G_{1}\left(x+v_{l} t\right)=\frac{1}{2 c} v_{r} \varphi\left(x+v_{l} t\right)-\frac{1}{2 c} \int_{x+v_{l} t}^{l_{0}} \psi(\xi) \mathrm{d} \xi-K,\left(0 \leq x \leq l_{0}-v_{l} t\right)
\end{aligned}
$$

The reflected waves are $G_{2}\left(x+v_{l} t\right)$ and $F_{2}\left(x-v_{r} t\right)$. Using the boundary conditions in Eq. (7), one has

$$
\left\{\begin{array}{c}
F_{1}\left(l_{0}-v_{r} t\right)+G_{2}\left(l_{0}+v_{l} t\right)=0 \\
G_{1}\left(v_{l} t\right)+F_{2}\left(-v_{r} t\right)=0
\end{array}\right.
$$

Defining $\alpha=l_{0}+v_{l} t, \beta=-v_{r} t$ and substituting $\alpha$ and $\beta$ into Eq. (10), one has 


$$
\left\{\begin{array}{c}
F_{2}(\beta)=-G_{1}\left(-\beta \frac{v_{l}}{v_{r}}\right) \\
G_{2}(\alpha)=-F_{1}\left(\frac{2 c l_{0}}{v_{l}}-\frac{v_{r}}{v_{l}} \alpha\right)
\end{array}\right.
$$

Using Eqs. (8), (9) and (11), the detailed expressions for $F_{2}$ and $G_{2}$ are

$$
\left\{\begin{array}{c}
F_{2}\left(x-v_{r} t\right)=-\frac{v_{r}}{2 c} \varphi\left(v_{l} t-\frac{v_{l}}{v_{r}} x\right)+\frac{1}{2 c} \int_{v_{l} t-\frac{v_{l}}{v_{r}} x}^{l_{0}} \psi(\xi) \mathrm{d} \xi-K \\
\left(0 \leq x \leq v_{r} t\right) \\
G_{2}\left(x+v_{l} t\right)=-\frac{v_{l}}{2 c} \varphi\left(\frac{2 c l_{0}}{v_{l}}-\frac{v_{r}}{v_{l}} x-v_{r} t\right)-\frac{1}{2 c} \int_{\frac{2 c l_{0}}{v_{l}}-\frac{v_{r}}{v_{l}} x-v_{r} t}^{l_{l}} \psi(\xi) \mathrm{d} \xi+K \\
\left(l_{0}-v_{l} t \leq x \leq l_{0}\right)
\end{array}\right.
$$

Combining $G_{1}, G_{2}$ and $F_{1}, F_{2}$, one has the expressions for $F$ and $G$, namely,

$$
\begin{aligned}
& F\left(x-v_{r} t\right)= \begin{cases}F_{1}\left(x-v_{r} t\right), & \left(v_{r} t \leq x \leq l_{0}\right) \\
F_{2}\left(x-v_{r} t\right), & \left(0 \leq x \leq v_{r} t\right)\end{cases} \\
& G\left(x+v_{l} t\right)= \begin{cases}G_{1}\left(x+v_{l} t\right), & \left(0 \leq x \leq l_{0}-v_{l} t\right) \\
G_{2}\left(x+v_{l} t\right), & \left(l_{0}-v_{l} t \leq x \leq l_{0}\right)\end{cases}
\end{aligned}
$$

Finally, the string displacement $w(x, t)$ in the interval $0 \leq t \leq t_{a}$ is given by

$$
w(x, t)=F\left(x-v_{r} t\right)+G\left(x+v_{l} t\right),\left(0 \leq x \leq l_{0}, 0 \leq t \leq t_{a}\right)
$$

3.1.2. The second time interval: $t_{a} \leq t \leq t_{b}$

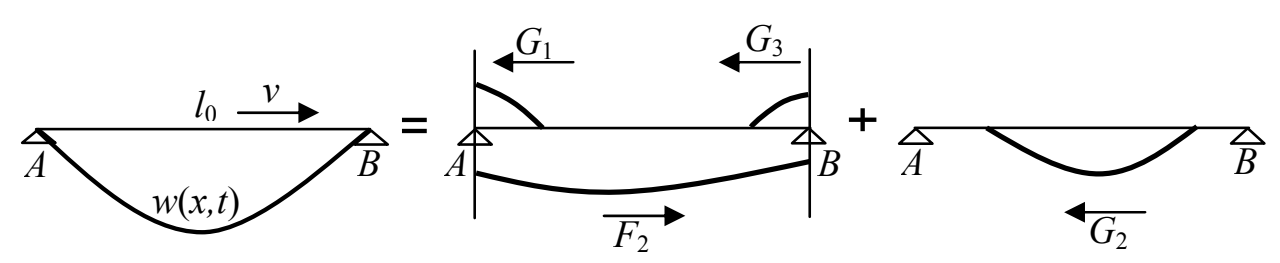

(a)

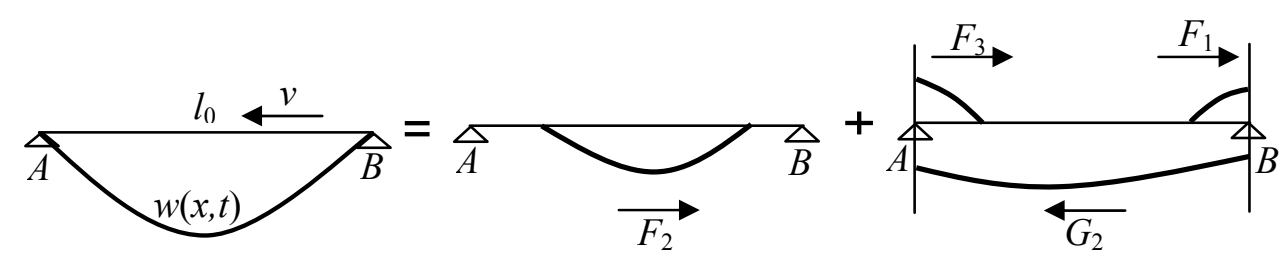

(b)

Fig. 3. String deflection comprising the sum of the propagating waves when $t_{a} \leq t \leq t_{b}$. The travelling speed of the string is positive $(v>0)$ for (a) and negative $(v<0)$ for (b).

In this time interval, if $v>0$, the waves are shown in Fig. 3(a). One can see that $F_{1}$ disappears and $G_{3}$ is the reflected wave of $F_{2}$ at $x=l_{0}$. Using the boundary condition (7), one has 


$$
G_{3}(x)=-F_{2}\left(l_{0}-v_{r} \frac{x-l_{0}}{v_{l}}\right)
$$

so

$$
G_{3}\left(x+v_{l} t\right)=\frac{v_{r}}{2 c} \varphi\left(x+v_{l} t-\frac{2 c l_{0}}{v_{r}}\right)-\frac{1}{2 c} \int_{x+v_{l} t-\frac{2 l_{0}}{v_{r}}}^{l_{0}} \psi(\xi) \mathrm{d} \xi-K,\left(\frac{2 c l_{0}}{v_{r}}-v_{l} t \leq x \leq l_{0}\right)
$$

If $v<0$, the waves are shown in Fig. 3(b). One can see that wave $G_{1}$ disappears and $F_{3}$ is the reflected wave of $G_{2}$ at $x=0$. Using the boundary condition Eq.(7), one has

$$
F_{3}(x)=-G_{2}\left(-\frac{v_{l}}{v_{r}} x\right)
$$

so

$$
F_{3}\left(x-v_{r} t\right)=\frac{v_{l}}{2 c} \varphi\left(x-v_{r} t+\frac{2 c l_{0}}{v_{l}}\right)+\frac{1}{2 c} \int_{x-v_{r} t+\frac{2 l_{0}}{v_{l}}}^{l_{0}} \psi(\xi) \mathrm{d} \xi+K,\left(0 \leq x \leq v_{r} t-\frac{v_{r}}{v_{l}} l_{0}\right)
$$

Combining $G_{1}, G_{2}, G_{3}$ and $F_{1}, F_{2}, F_{3}$, one has the combined wave expressions for $F$ and $G$,

$$
\begin{gathered}
F\left(x-v_{r} t\right)= \begin{cases}F_{2}\left(x-v_{r} t\right),\left(0 \leq x \leq l_{0},\right. & v>0) \\
F_{3}\left(x-v_{r} t\right),\left(0 \leq x \leq v_{r} t-\frac{v_{r}}{v_{l}} l_{0},\right. & v<0) \\
F_{2}\left(x-v_{r} t\right),\left(v_{r} t-\frac{v_{r}}{v_{l}} l_{0} \leq x \leq v_{r} t,\right. & v<0) \\
F_{1}\left(x-v_{r} t\right),\left(v_{r} t \leq x \leq l_{0},\right. & v<0)\end{cases} \\
G\left(x+v_{l} t\right)= \begin{cases}G_{1}\left(x+v_{l} t\right),\left(0 \leq x \leq l_{0}-v_{l} t,\right. & v>0) \\
G_{2}\left(x+v_{l} t\right),\left(l_{0}-v_{l} t \leq x \leq \frac{2 c l_{0}}{v_{r}}-v_{l} t,\right. & v>0) \\
G_{3}\left(x+v_{l} t\right),\left(\frac{2 c l_{0}}{v_{r}}-v_{l} t \leq x \leq l_{0},\right. & v>0) \\
G_{2}\left(x+v_{l} t\right),\left(0 \leq x \leq l_{0},\right. & v<0)\end{cases}
\end{gathered}
$$

Finally, the string displacement $w(x, t)$ in the interval $t_{a} \leq t \leq t_{b}$ is given by

$$
w(x, t)=F\left(x-v_{r} t\right)+G\left(x+v_{l} t\right),\left(0 \leq x \leq l_{0}, t_{a} \leq t \leq t_{b}\right)
$$

3.1.3. The third time interval: $t_{b} \leq t \leq T_{0}$

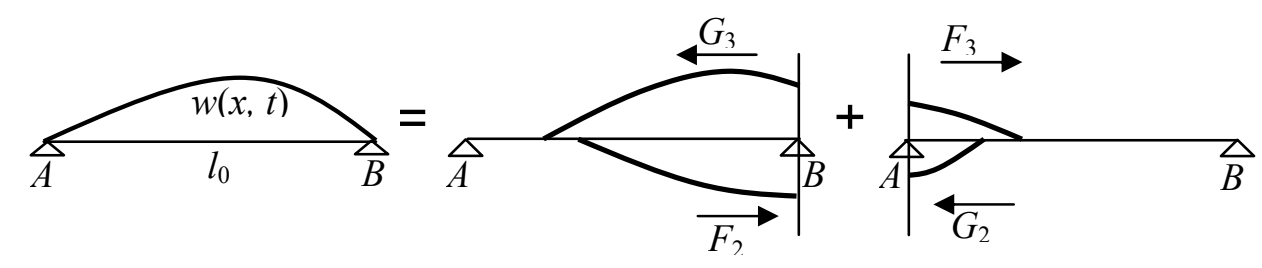


Fig. 4. String deflection comprising the sum of the propagating waves when $t_{b} \leq t \leq T_{0}$

In this time interval, the propagating waves are shown in Fig.4. $G_{3}$ is the reflected wave of $F_{2}$ at the boundary $x=l_{0}$ and $F_{3}$ is the reflected wave of $G_{2}$ at $x=0$. Combining $F_{2}, F_{3}$ and $G_{2}, G_{3}$, one has the expressions for $F$ and $G$,

$$
\begin{aligned}
& F\left(x-v_{r} t\right)=\left\{\begin{array}{l}
F_{2}\left(x-v_{r} t\right),\left(v_{r} t-\frac{v_{r}}{v_{l}} l_{0} \leq x \leq l_{0}\right) \\
F_{3}\left(x-v_{r} t\right),\left(0 \leq x \leq v_{r} t-\frac{v_{r}}{v_{l}} l_{0}\right)
\end{array}\right. \\
& G\left(x+v_{l} t\right)=\left\{\begin{array}{l}
G_{2}\left(x+v_{l} t\right),\left(0 \leq x \leq \frac{2 c l_{0}}{v_{r}}-v_{l} t\right) \\
G_{3}\left(x+v_{l} t\right),\left(\frac{2 c l_{0}}{v_{r}}-v_{l} t \leq x \leq l_{0}\right)
\end{array}\right.
\end{aligned}
$$

So the string displacement $w(x, t)$ in the time interval $t_{b} \leq t \leq T_{0}$ is given by

$$
w(x, t)=F\left(x-v_{r} t\right)+G\left(x+v_{l} t\right),\left(0 \leq x \leq l_{0}, t_{b} \leq t \leq T_{0}\right)
$$

\subsubsection{Continuity and smoothness}

It is necessary to prove that the vibrational response $w(x, t)$ exhibits both continuity and smoothness, since it is composed of piecewise functions. The procedure for establishing this check is given below.

Take the first time interval $\left(0 \leq t \leq t_{a}\right)$ for example. Because $G_{2}$ and $F_{2}$ are the reflected waves of $F_{1}$ and $G_{1}$, respectively, if $F_{1}$ and $F_{2}$ at the piecewise point $x=0$ as well as $G_{1}$ and $G_{2}$ at the piecewise point $x=l_{0}$ meet the continuity and smoothness when $t=0, w(x, 0)$ is continuous and smooth. Thus $w(x, t)$ is also continuous and smooth for it is just the superposition of the string deflections in the two wave components $F(x)$ and $G(x)$.

From Eq. (2), the initial conditions Eq. (3) and the boundary conditions Eq. (7), one can obtain

$$
\left\{\begin{array}{c}
F_{1}(0)+G_{1}(0)=w(0,0)=0 \\
F_{1}\left(l_{0}\right)+G_{1}\left(l_{0}\right)=w\left(l_{0}, 0\right)=0
\end{array}\right.
$$

Then from Eq. (10), one has

$$
\left\{\begin{array}{l}
F_{2}(0)=-G_{1}(0) \\
G_{2}\left(l_{0}\right)=-F_{1}\left(l_{0}\right)
\end{array}\right.
$$

Using Eqs. (26) and (27), the following equation is obtained, that is, the continuity condition: 


$$
\left\{\begin{array}{l}
F_{1}(0)=F_{2}(0) \\
G_{1}\left(l_{0}\right)=G_{2}\left(l_{0}\right)
\end{array}\right.
$$

So the response $w(x, 0)$ is continuous at the piecewise points $x=0$ and $x=l_{0}$. Hence, for the first time interval $\left(0 \leq t \leq t_{a}\right), w(x, t)$ is continuous also.

As for the smoothness, taking the $n$-order derivatives of $F_{1}(x), G_{1}(x), F_{2}(x)$ and $G_{2}(x)$ in Eqs. (8), (9) and (12) with respect to $x,(n=1,2,3, \ldots)$, one has

$$
\begin{gathered}
F_{1}^{(n)}(x)=\frac{v_{l}}{2 c} \varphi^{(n)}(x)-\frac{1}{2 c} \psi^{(n-1)}(x) \\
F_{2}^{(n)}(x)=-\frac{v_{r}}{2 c}\left(-\frac{v_{l}}{v_{r}}\right)^{n} \varphi^{(n)}\left(-\frac{v_{l}}{v_{r}} x\right)-\frac{1}{2 c}\left(-\frac{v_{l}}{v_{r}}\right)^{n} \psi^{(n-1)}\left(-\frac{v_{l}}{v_{r}} x\right) \\
G_{1}^{(n)}(x)=\frac{v_{r}}{2 c} \varphi^{(n)}(x)+\frac{1}{2 c} \psi^{(n-1)}(x) \\
G_{2}^{(n)}(x)=-\frac{v_{l}}{2 c}\left(-\frac{v_{r}}{v_{l}}\right)^{n} \varphi^{(n)}\left(\frac{2 c l_{0}}{v_{l}}-\frac{v_{r}}{v_{l}} x\right)+\frac{1}{2 c}\left(-\frac{v_{r}}{v_{l}}\right)^{n} \psi^{(n-1)}\left(\frac{2 c l_{0}}{v_{l}}-\frac{v_{r}}{v_{l}} x\right)
\end{gathered}
$$

where $\varphi^{(n)}(x)$ and $\psi^{(n)}(x)$ are the $n$-order derivatives of $\varphi(x)$ and $\psi(x)$ with respect to $x$. Substituting $x=0$ into Eq. (29) as well as $x=l_{0}$ into Eq. (30), then using the $n$-order smoothing condition at $x=0$ as well as $x=l_{0}$, one has

$$
\left\{\begin{array}{l}
F_{1}^{(n)}(0)=F_{2}^{(n)}(0) \\
G_{1}^{(n)}(0)=G_{2}^{(n)}(0)
\end{array}\right.
$$

The relationships which $\varphi(x)$ and $\psi(x)$ need to satisfy are obtained

$$
\left\{\begin{array}{l}
{\left[v_{l} v_{r}^{n}+v_{r}\left(-v_{l}\right)^{n}\right] \varphi^{(n)}(0)=\left[v_{r}^{n}-\left(-v_{l}\right)^{n}\right] \psi^{(n-1)}(0)} \\
{\left[v_{r} v_{l}^{n}+v_{l}\left(-v_{r}\right)^{n}\right] \varphi^{(n)}\left(l_{0}\right)=\left[\left(-v_{r}\right)^{n}-v_{l}^{n}\right] \psi^{(n-1)}\left(l_{0}\right)}
\end{array}\right.
$$

Substituting $n=1$ into Eq. (32), then 1-order smoothness is satisfied if

$$
\psi(0)=\psi\left(l_{0}\right)=0
$$

Also, 2-order smoothness $(n=2)$ is satisfied if

$$
\left\{\begin{array}{l}
\left(c^{2}-v^{2}\right) \varphi^{(2)}(0)=2 v \psi^{\prime}(0) \\
\left(c^{2}-v^{2}\right) \varphi^{(2)}\left(l_{0}\right)=2 v \psi^{\prime}\left(l_{0}\right)
\end{array}\right.
$$

As for $t_{a} \leq t \leq t_{b}$ and $t_{b} \leq t \leq T_{0}$, the same results for the continuity and smoothness as those shown in the initial time interval $0 \leq t \leq t_{a}$ can also be obtained.

\subsubsection{Numerical simulations for the fixed-fixed boundary case}

Taking into account the continuity and the first order smoothness, the initial conditions are chosen to be: 


$$
\left\{\begin{array}{c}
w(x, 0)=\varphi(x)=\left[H(x)-H\left(x-l_{0}\right)\right] A_{0} \sin ^{2}\left(\frac{\pi x}{l_{0}}\right) \\
w_{t}(x, 0)=\psi(x)=0
\end{array}\right.
$$

where $H$ is the Heaviside step function and $A_{0}$ is the initial displacement of the midpoint of the string which is chosen to be $A_{0}=0.05 \mathrm{~m}$. The constant length of the string is $l_{0}=5 \mathrm{~m}$. In order to verify the validity and accuracy of the proposed method, the results obtained are compared with that of other numerical solutions of arbitrary choice, such as the Newmark-Beta method and the state space function method used in previous numerical simulations in [11], which are approximate numerical solution methods. The results presented are shown in dimensionless form, where the displacement is presented as a proportion of the amplitude of the initial maximum displacement $A_{0}$, the axial coordinate as a proportion of the length of the string $l_{0}$ and the dimensionless travelling speed of string $V$ as a proportion of the critical velocity (namely, the free wave propagation speed c.), i.e. $V=v / c$.

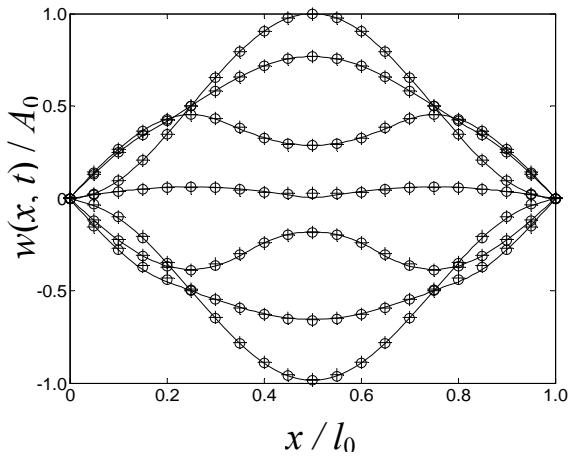

(a)

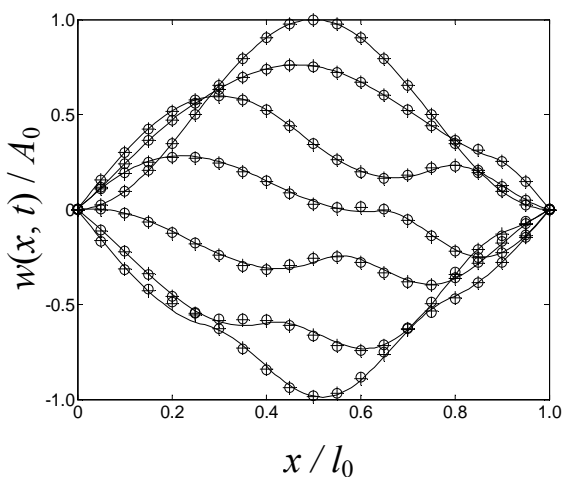

(c)

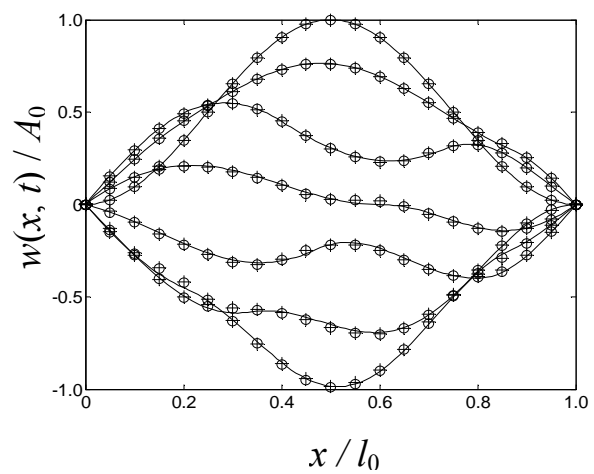

(b)

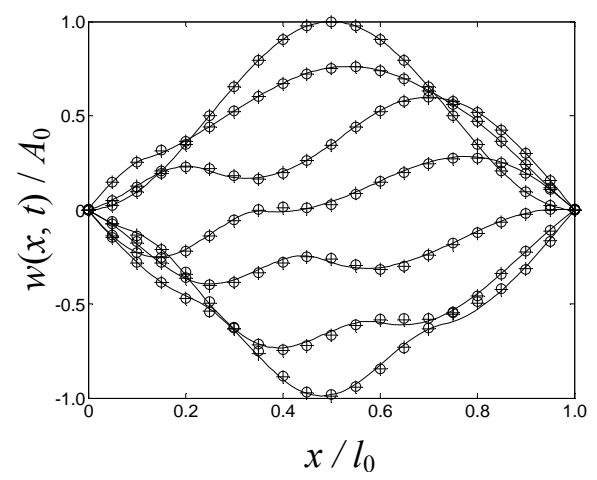

(d) 


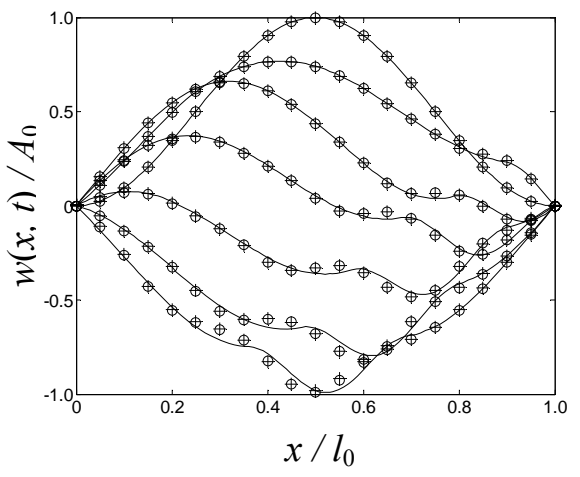

(e)

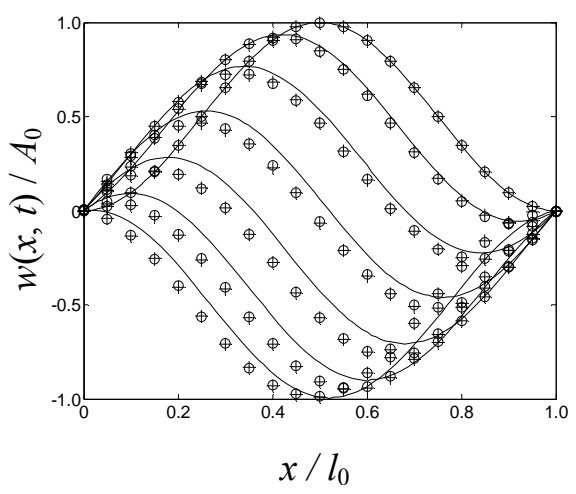

(f)

Fig. 5. Normalized string deflection shapes for an axially travelling string for the fixed-fixed boundary case with a constant distance between the boundaries. The non-dimensional string translational velocities $V=v / c$ and the minimum cycle $T_{0}$ required to return to the initial state are (a) $V=0, T_{0}=3.478 \mathrm{~s}$, (b) $V=0.2, T_{0}=3.296 \mathrm{~s}$, (c) $V=0.3, T_{0}=3.478 \mathrm{~s}$, (d) $V=-0.3, T_{0}=3.478 \mathrm{~s}$, (e) $V=0.5, T_{0}=4.219 \mathrm{~s}$, (f) $V=0.99, T_{0}=159.023 \mathrm{~s}$. Key: $\longrightarrow$, the proposed method; +++ , the Newmark-Beta method; o o o the state space function method

The results for the string displacement for the fixed-fixed case with constant length between the boundaries for six axial velocities are shown in Fig. 5. The seven curves from top to bottom in each plot are at the times corresponding to $0, T_{0} / 12$, $T_{0} / 6, T_{0} / 4, T_{0} / 3,5 T_{0} / 12$ and $T_{0} / 2$, respectively, where the minimum period $T_{0}$ is a function of the axially travelling velocity $v$ of the string (see Eq. (6)). The results obtained by the three methods are basically consistent at low translational velocities, i.e. $V \leq 0.5$, in Fig. 5 (a) to (e). With the string translational velocity increasing towards to the critical value $c$, i.e. $V=0.99$, as shown in Fig. 5 (f), the results of the Newmark-Beta method and the state space function method are increasingly more different than the analytical solution obtained by the proposed method as time increases. The results from the Newmark-Beta method and the state space function method are only approximate numerical solutions, which are affected by the ill conditioned matrix and cumulative errors etc., when the translational speed and the time increase. In contrast, the proposed method gives the exact analytical solutions so it is more stable than other two methods when the translational speed is close to the critical speed. One can see that Fig. 5(c) is the mirror image of Fig. 5(d) due to the equal and opposite travelling speeds $(V= \pm 0.3)$ of the string.

As for the computational efficiency as shown in Table 1, the proposed method is superior to the other two methods which run on a ThinkPad (type x240s) computer with an i7 processor using the Matlab R2012a software. When the travelling speed increasing towards to the critical speed $c$, i.e. $V=0.99$, the time taken by the Newmark-Beta method increases significantly, while the time taken by the proposed 
method remains small.

Table 1. Computational time comparison of the three methods (Unit: second)

\begin{tabular}{ccccccc}
\hline Methods & $V=0$ & $V=0.2$ & $V=0.3$ & $V=-0.3$ & $V=0.5$ & $V=0.99$ \\
\hline Newmark-Beta & 0.59 & 0.59 & 0.61 & 0.65 & 0.84 & 49.13 \\
State space function & 1.15 & 1.16 & 1.19 & 1.19 & 2.34 & 7.31 \\
Proposed method & 0.59 & 0.60 & 0.63 & 0.62 & 0.62 & 0.69 \\
\hline
\end{tabular}

\subsection{Fixed-free case}

In this case, the fixed support of the string at $x=0$ and the free support at $x=l_{0}$ are represented by the boundary conditions:

$$
\left\{\begin{array}{c}
w(0, t)=0 \\
w_{x}\left(l_{0}, t\right)=0
\end{array}\right.
$$

where $w_{x}$ is the first order partial derivation of $w(x, t)$ with respect to $x$. It is worth mentioning that the tension $T$ of the string can be maintained via the forces acting at two ends of the string. It is obvious that the waves $G_{2}, G_{3}$ and $F_{3}$ are different from the ones in section 3.1 for a free boundary condition at $x=l_{0}$. The string displacement $w(x, t)$ can be obtained by using the same method presented in section 3.1, just replacing the expressions for $G_{2}, G_{3}$ and $F_{3}$ with the new ones. These new expressions for $G_{2}, G_{3}$ and $F_{3}$ are considered in the follow sections.

\subsubsection{Wave $G_{2}$}

As shown in Fig. 2, $G_{2}$ is the reflection of $F_{1}$ at the free boundary, i.e. at $x=l_{0}$. Using the boundary conditions (36), one has

$$
G_{2}^{\prime}\left(l_{0}+v_{l} t\right)+F_{1}^{\prime}\left(l_{0}-v_{r} t\right)=0
$$

where $G_{2}^{\prime}$ is the first order derivative of $G_{2}(\gamma)$ versus $\gamma$, and the rest are the same.

Substituting $\alpha=l_{0}+v_{l} t$ into Eq. (37) yields

$$
G_{2}^{\prime}(\alpha)=-F_{1}^{\prime}\left(\frac{2 c l_{0}}{v_{l}}-\frac{v_{r}}{v_{l}} \alpha\right)
$$

Integrating Eq. (38) with respect to $\alpha$, one has

$$
\int_{x}^{l_{0}} G_{2}^{\prime}(\alpha) \mathrm{d} \alpha=\int_{x}^{l_{0}}-F_{1}^{\prime}\left(\frac{2 c l_{0}}{v_{l}}-\frac{v_{r}}{v_{l}} \alpha\right) \mathrm{d} \alpha
$$

then 


$$
G_{2}(x)=\frac{v_{l}}{v_{r}} F_{1}\left(\frac{2 c l_{0}}{v_{l}}-\frac{v_{r}}{v_{l}} x\right)+G_{2}\left(l_{0}\right)-\frac{v_{l}}{v_{r}} F_{1}\left(\frac{2 c l_{0}}{v_{l}}-\frac{v_{r}}{v_{l}} l_{0}\right)
$$

In order to have a continuous solution, an extra continuity condition has to be introduced, namely

$$
G_{1}\left(l_{0}, 0\right)=G_{2}\left(l_{0}, 0\right)
$$

Substituting Eqs. (8), (9) and (41) into Eq. (40), an expression for $G_{2}$ is obtained

$$
G_{2}\left(x+v_{l} t\right)=\frac{1}{2 c v_{r}}\left[\left(v_{r}{ }^{2}-v_{l}^{2}\right) \varphi\left(l_{0}\right)+v_{l}^{2} \varphi\left(\frac{2 c l_{0}}{v_{l}}-\frac{v_{r}}{v_{l}} x-v_{r} t\right)+v_{l} \int_{\frac{2 c l_{0}}{v_{l}}-\frac{v_{r}}{v_{l}} x-v_{r} t}^{l_{0}} \psi(\xi) \mathrm{d} \xi\right]-K
$$

\subsubsection{Waves $G_{3}$ and $F_{3}$}

As shown in Fig. 3(a), $G_{3}$ is the reflection of $F_{2}$ at $x=l_{0}$. Using the free boundary condition in Eq. (36), one has

$$
G_{3}^{\prime}\left(l_{0}+v_{l} t\right)=-F_{2}^{\prime}\left(l_{0}-v_{r} t\right)
$$

Integrating Eq. (43) with respect to $\alpha$ given in section 3.1.1, one has

$$
G_{3}(x)=G_{3}\left(l_{0}\right)+\frac{v_{l}}{v_{r}}\left[F_{2}\left(\frac{2 c l_{0}}{v_{l}}-\frac{v_{r}}{v_{l}} x\right)-F_{2}\left(l_{0}\right)\right]
$$

Considering the continuity condition, i.e.

$$
G_{3}\left(l_{0}, t_{a}\right)=G_{2}\left(l_{0}, t_{a}\right)
$$

and substituting Eqs. (12), (42) and (45) into Eq. (44), an expression for $G_{3}$ is obtained

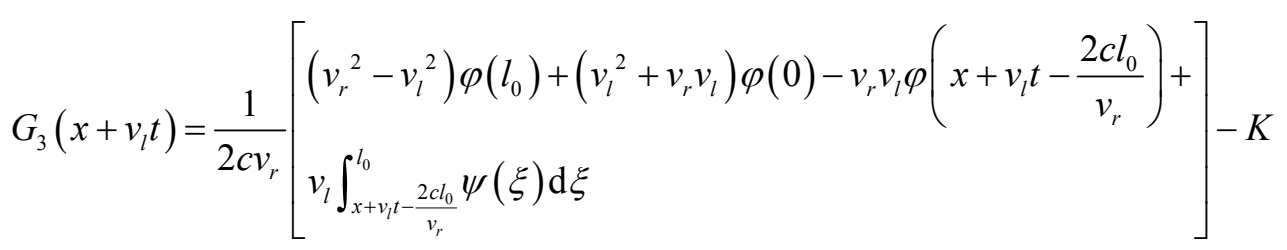

As shown in Fig. 3(b), $F_{3}$ is the reflection of $G_{2}$ at $x=0$. Using the fixed boundary condition at $x=0$ and Eq. (42), one has

$$
F_{3}\left(x-v_{r} t\right)=-\frac{1}{2 c v_{r}}\left[\left(v_{r}^{2}-v_{l}^{2}\right) \varphi\left(l_{0}\right)+v_{l}^{2} \varphi\left(x-v_{r} t+\frac{2 c l_{0}}{v_{l}}\right)+v_{l} \int_{x-v_{r} t+\frac{2 c l_{0}}{v_{l}}}^{l_{0}} \psi(\xi) \mathrm{d} \xi\right]-K
$$

\subsubsection{Continuity and smoothness}

As obtained from Eqs. (2), (40) and (41), a continuous solution for $w(x, t)$ is produced when the following additional condition for $\varphi$ at the free end $x=l_{0}$ is satisfied 


$$
\varphi\left(l_{0}\right)=0
$$

The $n$-order smoothness is satisfied if

$$
\left\{\begin{aligned}
{\left[v_{l} v_{r}^{n}+v_{r}\left(-v_{l}\right)^{n}\right] \varphi^{(n)}(0) } & =\left[v_{r}^{n}-\left(-v_{l}\right)^{n}\right] \psi^{(n-1)}(0) \\
{\left[v_{r} v_{l}^{n-1}+v_{l}\left(-v_{r}\right)^{n-1}\right] \varphi^{(n)}\left(l_{0}\right) } & =\left[\left(-v_{r}\right)^{n-1}-v_{l}^{n-1}\right] \psi^{(n-1)}\left(l_{0}\right)
\end{aligned}\right.
$$

When $n=1$, the additional condition required for smoothness is

$$
\varphi^{\prime}\left(l_{0}\right)=0
$$

and when $n=2$, the additional condition is

$$
\psi^{\prime}\left(l_{0}\right)=0
$$

\subsubsection{Numerical simulations for the fixed-free case}

The initial conditions are identical to as before and are also given by Eq. (35). The plot of the results are presented in Fig. 6 .

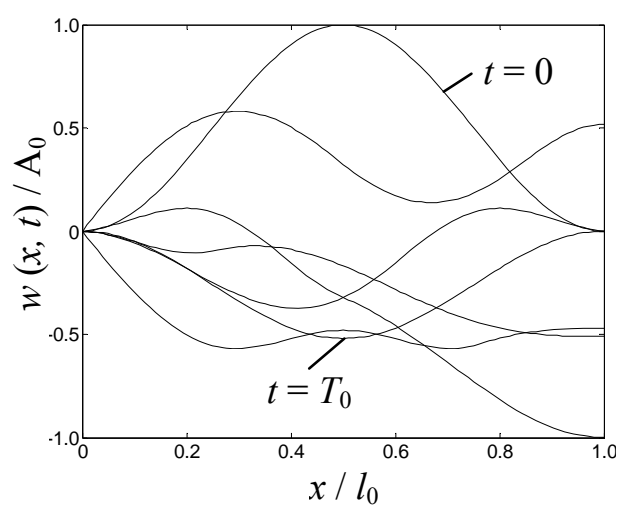

(a)

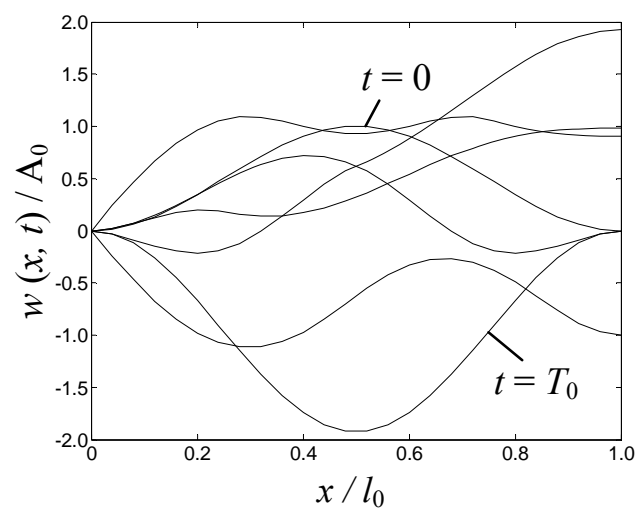

(c)

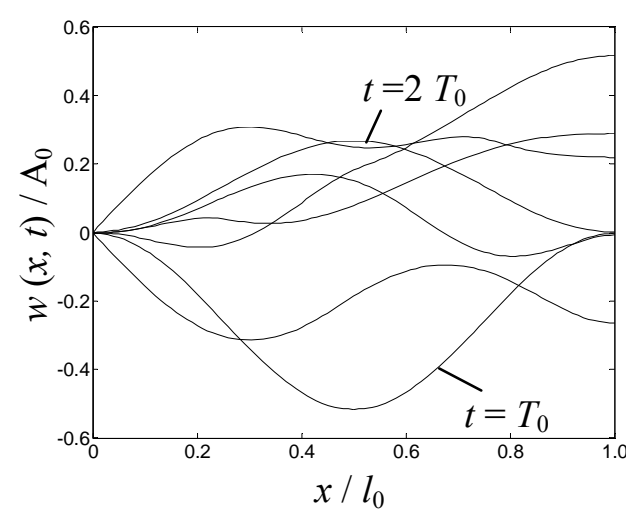

(b)

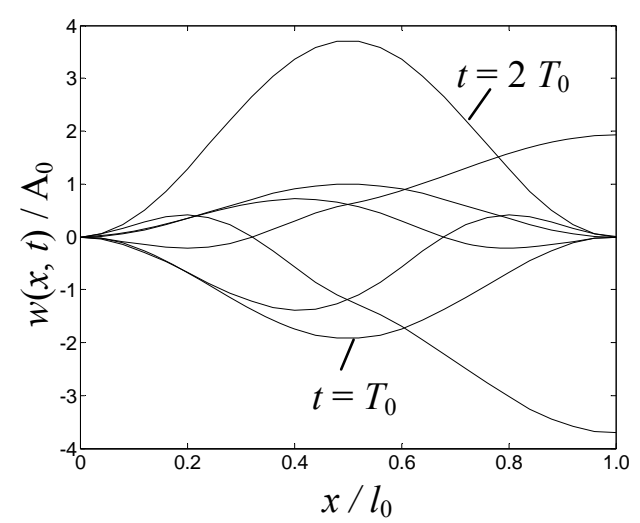

(d)

Fig. 6. Deflection shapes for an axially travelling string for the fixed-free boundary case with constant length and translational velocities for (a) $V=0.3$ in the first cycle, (b) $V=0.3$ in the second cycle, (c) $V=-0.3$ in the first cycle and (d) $V=-0.3$ in the second cycle.

The plots in Fig. 6 show the deflection shapes for an axially travelling string for 
the fixed-free boundary case with constant length over two cycles. The curves in Fig.6 (a) and Fig.6 (c) are in the first cycle, i.e. from top to bottom, and the ones in Fig.6 (b) and Fig.6 (d) are in the second cycle, i.e. from bottom to top. The time interval of adjacent curve is $T_{0} / 6$ in each plot. One can see that after one cycle the vibrational displacement of midpoint of the string has decreased when $V>0$, while increased when $V<0$.

\section{Travelling string with time-varying length}

In the time-varying length case, the length is assumed to change with time linearly, i.e. $l(t)=l_{0}+v t$. Defining $t_{n}$ as the time for the string to return to its original phase for the $n^{\text {th }}$ time, so the $n^{\text {th }}$ cycle is

$$
T_{n}=t_{n}-t_{n-1}
$$

In Eq. (52), define or choose $t_{0}=0$ when $n=1$. When $l(t)$ is a constant $l_{0}$, the $n^{\text {th }}$ cycle $T_{n}(n=1,2,3, \ldots)$ is also a constant value. When $l(t)$ is changing with time linearly, i.e. $l(t)=l_{0}+v t$, the $n^{\text {th }}$ cycle $T_{n}$ will change with $n$, as well as with time. The time varying cycle is calculated as follows.

\subsection{Time varying cycle $T_{n}$}

Defining $l_{n}$ as the length of string at the time of $t_{n}$, one can calculate $l_{n}$ as follows:

$$
l_{n}=l_{0}+v t_{n}
$$

The time varying cycle $T_{n}$ is actually equal to the time for the point $P_{2}$ in the right propagating wave $F$, shown in Fig.1, to pass through the string upstream and downstream in the $n^{\text {th }}$ cycle. For the upstream interval, the distance is $l_{n-1}$ and the propagating wave phase speed is $c-v$; while for the downstream interval, the distance is $l_{n-1}+v T_{n}$ and the propagating wave phase is $c+v$. Calculating the intervals corresponding to the upstream and downstream propagation yields

$$
\frac{l_{n-1}}{c-v}+\frac{l_{n-1}+v T_{n}}{c+v}=T_{n}
$$

Solving Eq. (54) yields the time varying cycle

$$
T_{n}=\frac{2 l_{n-1}}{c-v}
$$

Substitution of Eqs. (55) and (53) into Eq. (52) gives an iterative equation for $t_{n}$ as follows,

$$
\left\{\begin{array}{c}
t_{n}=\frac{2 l_{0}+(v+c) t_{n-1}}{c-v},(n=1,2,3, \ldots), \\
t_{0}=0
\end{array}\right.
$$

Now, substituting the results of Eq. (56) into Eq. (52), one can finally obtain the time varying cycle $T_{n},(n=1,2,3, \ldots)$ for the vibration of time varying length string. 


\subsection{Free vibration calculation}

For each cycle, the three time intervals $\left[0, t_{a}\right],\left[t_{a}, t_{b}\right]$ and $\left[t_{b}, T_{0}\right]$ defined in section 3 are changing in this time varying string length case. Defining $t_{a n}$ as the first piecewise point and $t_{b n}$ as the second piecewise point in the $n^{\text {th }}$ cycle, then the three time intervals in cycle $T_{n}$ are $\left[t_{n-1}, t_{a n}\right],\left[t_{a n}, t_{b n}\right]$ and $\left[t_{b n}, t_{n}\right]$. The values of $t_{a n}, t_{b n}$ and $t_{n}$ are given as follows

$$
\left\{\begin{array}{l}
t_{a n}=\frac{l_{n-1}}{c}+t_{n-1} \\
t_{b n}=\frac{l_{n-1}}{c-v}+t_{n-1},\left(v>0, t_{0}=0, n=1,2,3, \ldots\right) \\
t_{n}=\frac{2 l_{n-1}}{c-v}+t_{n-1}
\end{array}\right.
$$

Using Eqs. (56) and (57) one can calculate the values of $t_{a n}, t_{b n}$ and $t_{n}$ by the iterative method. However, for the case of $v<0$, i.e. the length of the string is shortened, the values of $t_{a n}$ and $t_{b n}$ exchange with each other, i.e. the three time intervals in cycle $T_{n}$ are $\left[t_{n-1}, t_{b n}\right],\left[t_{b n}, t_{a n}\right]$ and $\left[t_{a n}, t_{n}\right]$ when $v<0$. Finally, the response of the string with time varying length can be obtained using the expressions similar to those in Eqs. (15), (22) and (25), only needing to replace the three time intervals $\left[0, t_{a}\right],\left[t_{a}, t_{b}\right],\left[t_{b}, T_{0}\right]$ with the three new time intervals $\left[t_{n-1}, t_{b n}\right],\left[t_{b n}, t_{a n}\right]$ and $\left[t_{a n}, t_{n}\right]$ as well as the value $l_{0}$ with $l(t)$.

\subsection{Numerical simulations for a string with time-varying length}

In this case, the speed of the string is constant, yet the length is time-varying, i.e. the support of the string is fixed at $x=0$ while the opposite end moves with the same speed as the string at $x=l(t)$. The initial conditions are again given by Eq. (35). Fig. 7 (a) is the case of increasing length with $V=0.3$ and Fig. 7(b) is the case of decreasing length with $V=-0.3$.

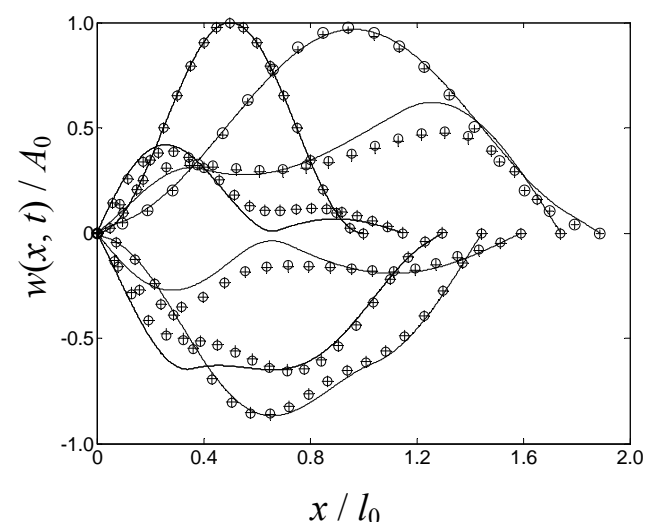

(a)

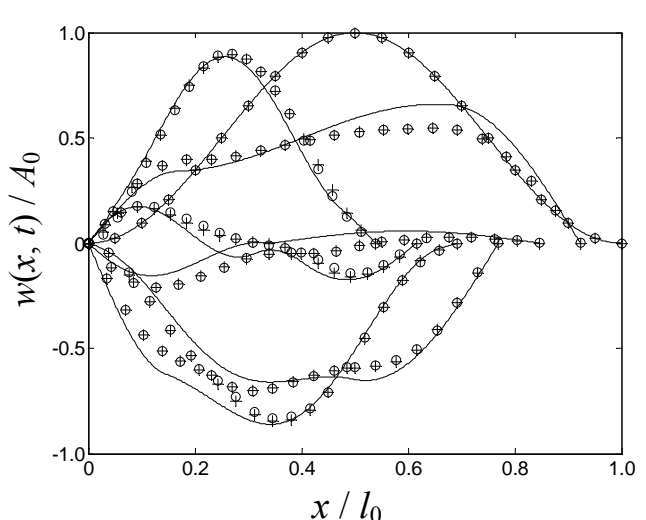

(b)

Fig. 7. Deflection shapes of an axially travelling string for the fixed-fixed case with time-varying length at axial velocities (a) $V=0.3$, (b) $V=-0.3$. Key: the proposed 
method; +++ , the Newmark-Beta method; o o o and the state space function method

The seven curves in each plot are at the corresponding times of $0, T_{1} / 6, T_{1} / 3, T_{1} / 2$, $2 T_{1} / 3,5 T_{1} / 6$ and $T_{1}$, respectively. $T_{1}$ is the time for first cycle, i.e. $T_{n}$ for $n=1$. One can see that the results of the analytical method proposed in this paper are in good agreement with those of the other two methods in the earliest time intervals. As time increases, the error of the approximate methods, i.e. the Newmark-Beta method and the state space function method, increase gradually and the results of the approximate methods are not in so good an agreement with that of the proposed method.

\section{Vibration energy analysis}

In this section, the total mechanical energy in the axially travelling string is analyzed based on the proposed reflected wave superposition method for the different types of boundary conditions and working conditions, as presented in sections 3 and 4 . The total mechanical energy $E(t)$ of the axially travelling string comprises the kinetic and potential energy in the following way [6]:

$$
E(t)=\frac{\rho}{2} \int_{0}^{l(t)}\left[\left(w_{t}+v w_{x}\right)^{2}+c^{2} w_{x}^{2}\right] \mathrm{d} x
$$

\subsection{Vibrational energy for a constant string length between the fixed-fixed}

\section{boundaries}

\subsubsection{The first time interval: $0 \leq t \leq t_{a}$}

As shown in Fig.2, the response of the string comprises four propagating waves $F_{1}$, $F_{2}, G_{1}$ and $G_{2}$, so the total energy of the string is the combination of the energy of these four propagating waves. The total energy expression can be written:

$$
E(t)=E_{F_{1}}(t)+E_{F_{2}}(t)+E_{G_{1}}(t)+E_{G_{2}}(t)
$$

where $E_{F_{1}}(t), E_{F_{2}}(t), E_{G_{1}}(t)$ and $E_{G_{2}}(t)$ are the energy of waves $F_{1}, F_{2}, G_{1}$ and $G_{2}$. According to Eq. (58), one can obtain energy expression of travelling waves $F_{1}$ :

$$
E_{F_{1}}(t)=\frac{\rho}{2} \int_{v_{r} t}^{l_{0}}\left[\left(-v_{r} F_{1}^{\prime}+v F_{1}^{\prime}\right)^{2}+c^{2} F_{1}^{\prime 2}\right] \mathrm{d} x=\rho c^{2} \int_{v_{r} t}^{l_{0}} F_{1}^{\prime 2} \mathrm{~d} x
$$

Similarly, the expressions of $E_{F_{2}}(t), E_{G_{1}}(t)$ and $E_{G_{2}}(t)$ are as follows.

$$
\left\{\begin{array}{l}
E_{F_{2}}(t)=\rho c^{2} \int_{0}^{v_{r} t} F_{2}^{\prime 2} \mathrm{~d} x \\
E_{G_{1}}(t)=\rho c^{2} \int_{0}^{l_{0}-v_{l} t} G_{1}^{\prime 2} \mathrm{~d} x \\
E_{G_{2}}(t)=\rho c^{2} \int_{l_{0}-v_{t} t}^{l_{0}} G_{2}^{\prime 2} \mathrm{~d} x
\end{array}\right.
$$

Substituting the expressions for the traveling waves $F_{1}, F_{2}, G_{1}$ and $G_{2}$, i.e. Eqs. (8), (9) and (12), into Eqs. (60) and (61) correspondingly, one can has the wave energies in 
the interval $0 \leq t \leq t_{a}$ shown as follows.

$$
\left\{\begin{array}{l}
E_{F_{1}}(t)=\frac{\rho v_{l}^{2}}{4} \int_{v_{r} t}^{l_{0}} \varphi^{2}\left(x-v_{r} t\right) \mathrm{d} x \\
E_{F_{2}}(t)=\frac{\rho v_{l}^{2}}{4} \int_{0}^{v_{r} t} \varphi^{\prime 2}\left(v_{l} t-\frac{v_{l}}{v_{r}} x\right) \mathrm{d} x \\
E_{G_{1}}(t)=\frac{\rho v_{r}{ }^{2}}{4} \int_{0}^{l_{0}-v_{l} t} \varphi^{2}\left(x+v_{l} t\right) \mathrm{d} x \\
E_{G_{2}}(t)=\frac{\rho v_{r}{ }^{2}}{4} \int_{l_{0}-v_{l} t}^{l_{0}} \varphi^{2}\left(\frac{2 c l_{0}}{v_{l}}-\frac{v_{r}}{v_{l}} x-v_{r} t\right) \mathrm{d} x
\end{array} \quad\left(0 t_{a}\right)\right.
$$

\subsubsection{The second time interval: $t_{a} \leq t \leq t_{b}$}

In this time interval, different expressions exist depending upon the direction in which the string is translating, i.e. for $v>0$ and $v<0$. When $v>0$, the energy is due to the waves $G_{1}, G_{2}, G_{3}$, and $F_{2}$,

$$
\left\{\begin{array}{l}
E_{G_{1}}(t)=\frac{\rho v_{r}^{2}}{4} \int_{0}^{l_{0}-v_{l} t} \varphi^{2}\left(x+v_{l} t\right) \mathrm{d} x \\
E_{G_{2}}(t)=\frac{\rho v_{r}^{2}}{4} \int_{l_{0}-v_{l} t}^{2 \frac{2 c_{0}}{v_{r}}-v_{l} t} \varphi^{\prime 2}\left(\frac{2 c l_{0}}{v_{l}}-\frac{v_{r}}{v_{l}} x-v_{r} t\right) \mathrm{d} x \\
E_{G_{3}}(t)=\frac{\rho v_{r}^{2}}{4} \int_{\frac{2 l_{0}}{v_{r}}-v_{l} t}^{l_{0}} \varphi^{\prime 2}\left(x+v_{l} t-\frac{2 c l_{0}}{v_{r}}\right) \mathrm{d} x \\
E_{F_{2}}(t)=\frac{\rho v_{l}^{2}}{4} \int_{0}^{l_{0}} \varphi^{\prime 2}\left(v_{l} t-\frac{v_{l}}{v_{r}} x\right) \mathrm{d} x
\end{array} \quad\left(v>0, t_{a} \leq t \leq t_{b}\right)\right.
$$

When $v<0$, the energy is due to the waves $F_{1}, F_{2}, F_{3}$, and $G_{2}$,

$$
\left\{\begin{array}{l}
E_{F_{1}}(t)=\frac{\rho v_{l}^{2}}{4} \int_{v_{r} t}^{l_{0}} \varphi^{\prime 2}\left(x-v_{r} t\right) \mathrm{d} x \\
E_{F_{2}}(t)=\frac{\rho v_{l}^{2}}{4} \int_{v_{r}, t-\frac{v_{r}}{v_{l}} l_{0}}^{v_{r} t} \varphi^{2}\left(v_{l} t-\frac{v_{l}}{v_{r}} x\right) \mathrm{d} x \\
E_{F_{3}}(t)=\frac{\rho v_{l}^{2}}{4} \int_{0}^{v_{r} t-\frac{v_{r}}{v_{l}} l_{0}} \varphi^{\prime 2}\left(x-v_{r} t+\frac{2 c l_{0}}{v_{l}}\right) \mathrm{d} x \\
E_{G_{2}}(t) \frac{\rho v_{r}^{2}}{4} \int_{0}^{l_{0}} \varphi^{2}\left(\frac{2 c l_{0}}{v_{l}}-\frac{v_{r}}{v_{l}} x-v_{r} t\right) \mathrm{d} x
\end{array}\right.
$$

\subsubsection{The third time interval: $t_{b} \leq t \leq T_{0}$}

In this interval, the energy is due to the waves $F_{2}, F_{3}, G_{2}$, and $G_{3}$. 


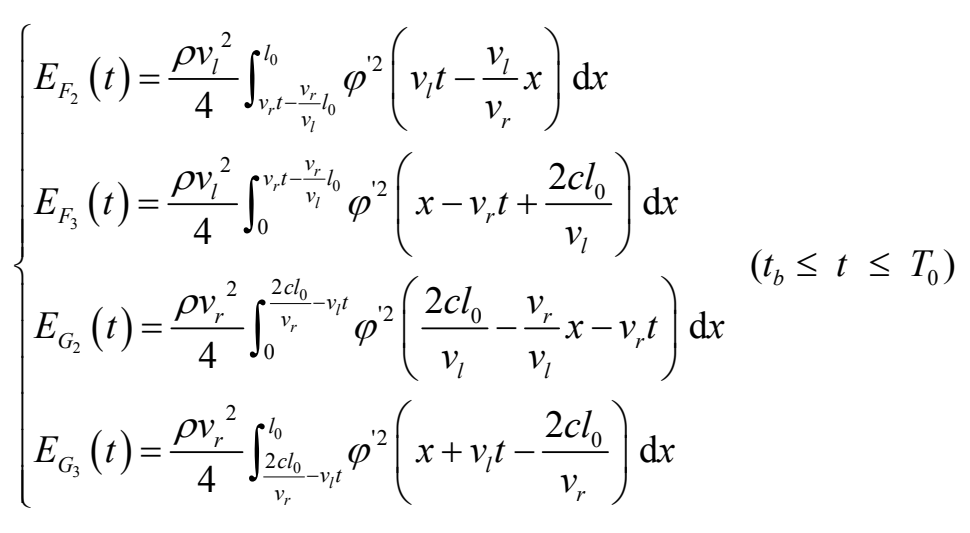

So the total mechanical energy of the axially travelling string can be calculated using Eqs. (62) - (65) and the initial conditions. The total energy of an axially travelling string with fixed-fixed boundary conditions and with $V=-0.5, V=0$, and $V=0.5$ for one cycle is shown in Fig. 8. In this case, the boundary conditions are symmetric, so the energy variations are the same when their velocities are equal in magnitude but in opposite directions. When $V=0$, the energy of the string is unchanged during one cycle $T_{0}$ and the corresponding string deflection shape is shown in Fig. 5(a), which is the standing wave. When $V= \pm 0.5$, the total energy of the string at the beginning and end of one cycle $T_{0}$ are equal. It shows that the total energy of the system varies with time due to energy transfer at both boundaries, so the string deflection shapes is not standing wave which are shown in Fig. 5(c) and (d). The string segment passing through a downstream fixed boundary gains energy, and it loses energy at an upstream one [15], while in a cycle $T_{0}$, the loss of energy is equal to the energy that is obtained for a constant length string with symmetric boundaries. One can easily speculate that the curve for the change in the energy in other cycles is the same as in the first cycle shown in Fig.8.

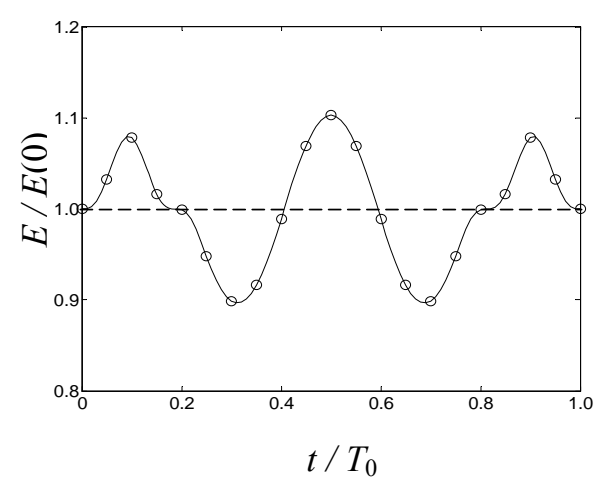

Fig. 8. Energy variation of the lateral vibration in an axially travelling string. The interval of free motion is $T_{0}=2.667 \mathrm{~s}$ and the string is fixed at its two ends with constant length.

Key: o o o, $V=0.5,---, V=0,-, V=0.5$.

5.2. Vibrational energy for a constant string length between asymmetric boundary configurations 
The energy in two cycles will be studied for the case of asymmetric boundary configurations, i.e. the fixed-free case and the free-fixed case. The calculation process for the energy expression for the waves in the second cycle is similar to the one in the first cycle. It is obvious that the energy expression for the waves $G_{2}, G_{3}$ and $F_{3}$ are different from the ones in section 5.1 for a free boundary condition at $x=l_{0}$. The energy can be obtained using the same method presented in section 5.1, just replacing the energy expressions for $G_{2}, G_{3}$ and $F_{3}$ with the new ones. Take the third time interval $\left(t_{b} \leq t \leq T_{0}\right)$ for example. Replacing $F_{1}$ in Eq. (60) with $G_{2}, G_{3}, F_{2}$ and $F_{3}$, which are expressed in Eqs. (42), (46), (12) and (47), respectively, one can has the new energy expressions for $G_{2}, G_{3}, F_{2}$ and $F_{3}$ in this time interval.

$$
\left\{\begin{array}{l}
E_{G_{2}}(t)=\frac{\rho v_{l}^{2}}{4} \int_{0}^{\frac{2 c l_{0}}{v_{r}}-v_{l} t} \varphi^{2}\left(\frac{2 c l_{0}}{v_{l}}-\frac{v_{r}}{v_{l}} x-v_{r} t\right) \mathrm{d} x \\
E_{G_{3}}(t)=\frac{\rho v_{l}^{2}}{4} \int_{\frac{2 c l_{0}}{v_{r}} v_{l} t}^{l_{0}} \varphi^{2}\left(x+v_{l} t-\frac{2 c l_{0}}{v_{r}}\right) \mathrm{d} x \\
E_{F_{2}}(t)=\frac{\rho v_{l}^{2}}{4} \int_{v_{r} t-\frac{v_{r}}{v_{l}} l_{0}}^{l_{0}} \varphi^{2}\left(v_{l} t-\frac{v_{l}}{v_{r}} x\right) \mathrm{d} x \\
E_{F_{3}}(t)=\frac{\rho v_{l}^{4}}{4 v_{r}^{2}} \int_{0}^{v_{r} t-\frac{v_{r}}{v_{l}} l_{0}} \varphi^{\prime 2}\left(x-v_{r} t+\frac{2 c l_{0}}{v_{l}}\right) \mathrm{d} x
\end{array} \quad\left(t_{b} \leq T_{0}\right)\right.
$$

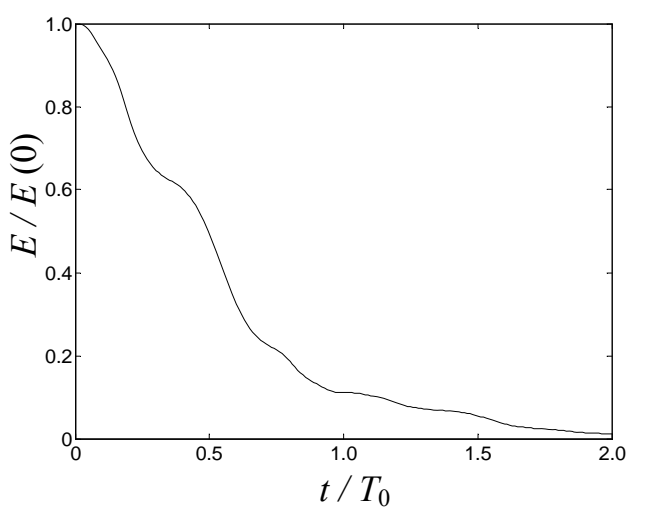

(a)

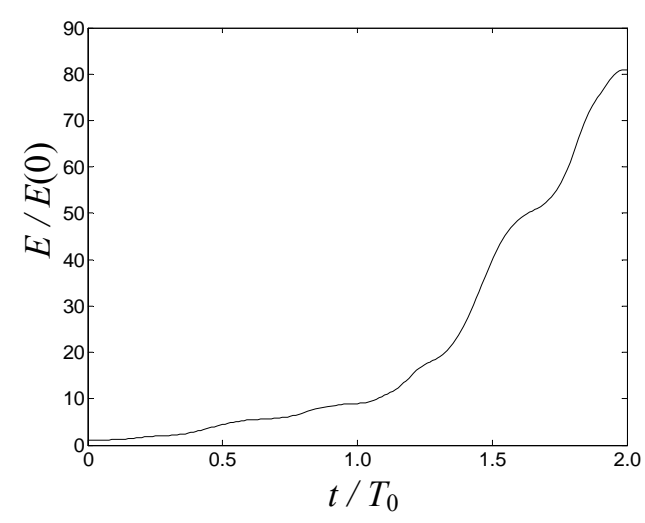

(b)

Fig. 9. Energy variation of the lateral vibration in an axially travelling string with asymmetric boundary conditions, which are (a) fixed-free case with $V=0.5$ and (b) equivalent to free-fixed case with $V=-0.5$.

Fig. 9 shows the change in the total vibrational energy curves over the first two cycles, i.e. $0<t<2 T_{0}$. In Fig. 9 (a), the energy is decreasing with time because energy flux is always transferred out of the system at the left end, i.e. the fixed boundaries, with the string travelling with a positive speed. The rate for the decrease in the energy reduces with time and the total energy is close to zero after two periods but never equal to zero. No energy exchange at the free end because there is no work done there. Fig. 9 (b) gives the contrary phenomenon, i.e. the energy is increasing with time because energy flux is always transferred into the system at the left fixed 
end. Asymmetric boundary configurations lead to dissipated ( $V=0.5$, fixed-free) or self-excited motions ( $V=-0.5$, free-fixed) for the free vibration of the axially travelling string, similar to what has been observed in a tensioned pipe[15].

\subsection{Vibrational energy for a varying length string between fixed-fixed boundaries}

In this case, the length is assumed to be changing with time linearly and the string is fixed at its two ends. As previously, the energy expression for the waves $G_{2}$, $G_{3}$ and $F_{3}$ are different from the ones in section 5.1 for a moving boundary condition at $x=l(\mathrm{t})$. Only the energy of first cycle will be studied, it means we take $n=1$ in Eq. (44). The energy expression for the waves $G_{2}, G_{3}$ and $F_{3}$ will be calculated in three time durations respectively and the calculation process is similar to the fixed-fixed case. Taking the third time interval $\left(t_{b 1} \leq t \leq T_{1}\right)$ for example, one has the corresponding energy expressions for the waves $F_{2}, F_{3}, G_{2}$ and $G_{3}$

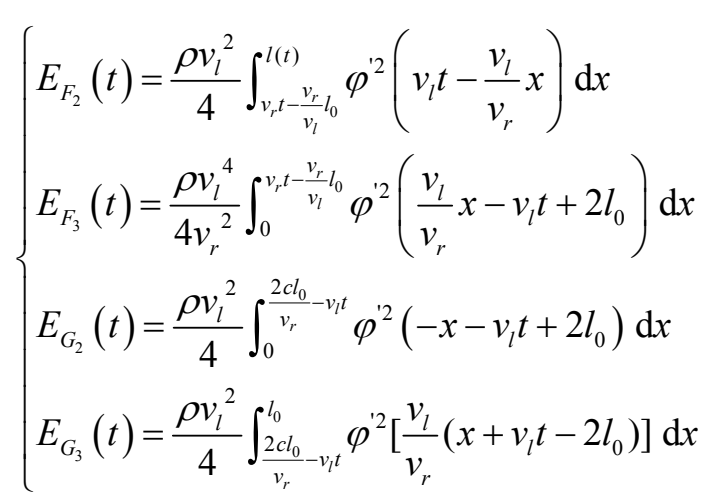

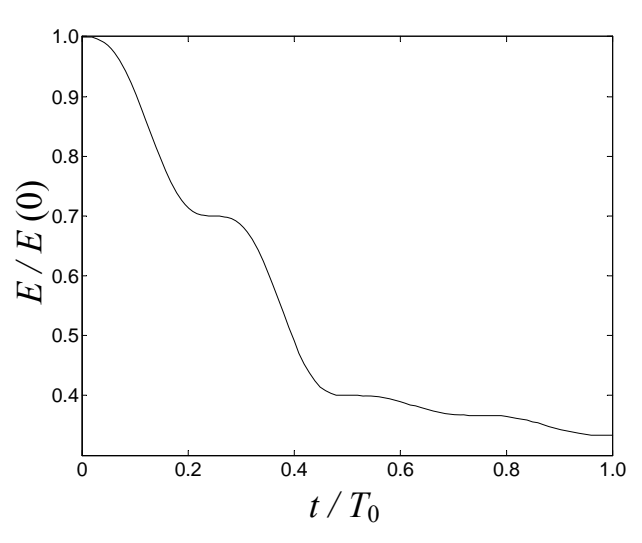

(a)

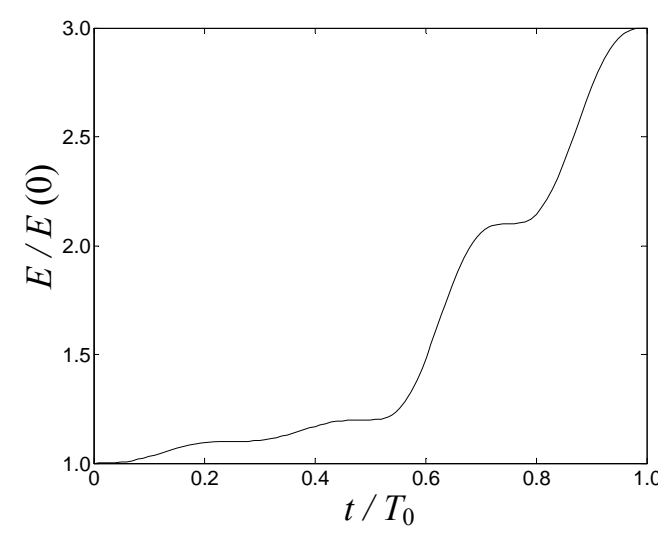

(b)

Fig. 10. Energy variation of the lateral vibration in an axially travelling string with time varying length. The dimensionless travelling speeds of string are (a) $V=0.5$ for increasing length case and (b) $V=-0.5$ for decreasing length case.

In Fig. 10, the energy variation with time is given for the travelling varying length string which is fixed in the transverse direction at the two ends. The energy decays in the case of an increasing string length in Fig. 10 (a) and grows in case of a decreasing length in Fig. 10 (b) over one period. Similar to Section 5.1, the string 
segment passing through a downstream fixed boundary gains energy, and it loses energy at an upstream fixed one. However, the moving end does no work to the string because it moves synchronously with the string, which is different from the fixed-fixed constant length case in Section 5.1. So the energy variation curves in Fig. 10 are similar to the ones in Fig. 9. When the string length increases, the static end support does negative work, consuming part of the vibrational energy of the system; while when the string length decreases, the static end support does positive work, increasing the total vibrational energy in the string system, which is called the spaghetti problem. Usually, a proper damper is placed at the static end of a decreasing length string to depress the vibration. The energy analytical calculation and analysis provide theoretical basis for vibration control of a string by damping.

\section{Conclusions}

A propagating and reflected wave superposition method is proposed in this paper to obtain the analytical solutions for the free vibration of an axially translating string. The string can be either of constant or time varying length and is subject to some initial string displacement conditions. From application of this method, one can consider and examine the physical nature of the string vibration based on the concept of wave propagation. For reasons of simplicity, only two types of classical boundary conditions are studied in this paper; while other nonclassical boundary conditions are also suitable for analysis using the same method. Compared with the Newmark-Beta method and the state space function method, the proposed method is very consistent with these two methods at low translational speeds, whilst being more stable and efficient than the other two methods at speeds close to the critical value. The total mechanical energy of a string has been calculated analytically based on the analytic vibration response via the proposed method for the different types of boundary conditions and working conditions, from which one can understand the mechanism of energy variation due to these boundaries. For the fixed-free and free-fixed boundary conditions, results show that the asymmetric boundary conditions have opposite contributions and effects on the total energy. For the time varying length string, the energy variation has similar characteristics and mechanism to the constant length fixed-free case. As a result, this analysis provides a basis for the vibration control of axially travelling string system by placing a passive vibration control mechanism at an optimum location.

\section{Acknowledgments}

This work was supported by the National Natural Science Foundation of China [grant numbers 51305115 and 51675150$]$. 


\section{Appendix A Derivation of the motion equation}

The motion equation of a travelling string will be obtained by applying the Hamilton's principle in the following form:

$$
\delta \int_{t_{2}}^{t_{1}} L \mathrm{~d} t=0
$$

where $\delta$ is a variation in a function, $L=K-U$ is the Lagrangian function which requires the kinetic energy $K$ given by

$$
K=\frac{1}{2} \int_{0}^{l(t)} \rho\left(w_{t}+v w_{x}\right)^{2} \mathrm{~d} x
$$

and the potential energy $U$ given by

$$
U=\frac{1}{2} \int_{0}^{l(t)} T w_{x}^{2} \mathrm{~d} x
$$

Substituting Eqs. (A2) and (A3) into Eq. (A1), one can obtain

$$
\frac{1}{2} \delta \int_{t_{2}}^{t_{1}} \int_{0}^{l(t)}\left[\rho\left(w_{t}^{2}+2 v w_{t} w_{x}+v^{2} w_{x}^{2}\right)-T w_{x}^{2}\right] \mathrm{d} x \mathrm{~d} t=0
$$

Taking the variation with respect to $w_{t}$ and $w_{x}$ in Eq. (A4), the expression becomes

$$
\int_{t_{2}}^{t_{1}} \int_{0}^{l(t)}\left\{\rho\left(w_{t}+v w_{x}\right) \delta w_{t}+\left[\rho\left(v w_{t}+v^{2} w_{x}\right)-T w_{x}\right] \delta w_{x}\right\} \mathrm{d} x \mathrm{~d} t=0
$$

Applying integration by parts to the two terms in the integral in the Eq. (A5), one can obtain

$$
\begin{aligned}
& \int_{0}^{l(t)} \rho\left(w_{t}+v w_{x}\right) \delta w_{t} \mathrm{~d} x=\frac{\partial}{\partial t} \int_{0}^{l(t)} \rho\left(w_{t}+v w_{x}\right) \delta w \mathrm{~d} x-\int_{0}^{l(t)} \rho\left(w_{t t}+v w_{x t}\right) \delta w \mathrm{~d} x \\
& \int_{0}^{l(t)}\left[\rho\left(v w_{t}+v^{2} w_{x}\right)-T w_{x}\right] \delta w_{x} \mathrm{~d} x=\frac{\partial}{\partial x} \int_{0}^{l(t)}\left[\rho\left(v w_{t}+v^{2} w_{x}\right)-T w_{x}\right] \delta w \mathrm{~d} x \\
& -\int_{0}^{l(t)}\left[\rho\left(v w_{x t}+v^{2} w_{x x}\right)-T w_{x x}\right] \delta w \mathrm{~d} x
\end{aligned}
$$

According to the Hamilton's principle, the time end conditions are satisfied, i.e.

$$
t=t_{1}, \delta w=0 ; t=t_{2}, \delta w=0
$$

Substituting Eqs. (A6), (A7) and (A8) into Eq. (A5) and collecting the terms, one can obtain

$$
\left.\int_{t_{1}}^{t_{2}}\left[\rho\left(v w_{t}+v^{2} w_{x}\right)-T w_{x}\right] \delta w\right|_{x=0} ^{x=l(t)} \mathrm{d} t-\int_{t_{1}}^{t_{2}} \int_{0}^{l(t)}\left[\rho\left(w_{t t}+2 v w_{x t}+v^{2} w_{x x}\right)-T w_{x x}\right] \delta w \mathrm{~d} x \mathrm{~d} t=0
$$

Since the variation $\delta w$ over the interval $0<x<l(t)$ is arbitrary, Eq. (A9) can be satisfied only when the individual terms of Eq. (A9) are equal to zero.

$$
\begin{gathered}
{\left.\left[\rho\left(v w_{t}+v^{2} w_{x}\right)-T w_{x}\right] \delta w\right|_{x=0} ^{x=l(t)}=0} \\
\rho\left(w_{t t}+2 v w_{x t}+v^{2} w_{x x}\right)-T w_{x x}=0
\end{gathered}
$$


Eq. (A10) represents the boundary condition while Eq. (A11) denotes the equation of motion. Substituting $c=\sqrt{T / \rho}$ into Eq. (A11), one can obtain

$$
w_{t t}+2 v w_{x t}+\left(v^{2}-c^{2}\right) w_{x x}=0
$$

\section{Appendix B Applicability of d'Alembert method in the travelling string equation}

The general solution of Eq. (1) in the form of moving wave is

$$
w(x, t)=f\left(x-c_{1} t\right)
$$

After partial derivatives of $w(x, t)$ with respect to $x$ and $t$, one obtains

$$
\begin{gathered}
w_{t t}=c_{1}^{2} f^{\prime \prime}\left(x-c_{1} t\right) \\
w_{t x}=-c_{1} f^{\prime \prime}\left(x-c_{1} t\right) \\
w_{x x}=f^{\prime \prime}\left(x-c_{1} t\right)
\end{gathered}
$$

Substituting Eqs. (B2), (B3) and (B4) into Eq. (1), one has

$$
c_{1}^{2}-2 v c_{1}+v^{2}-c^{2}=0
$$

Then $c_{1}$ is obtained as follow

$$
c_{1}=v+c \text { or } c_{1}=v-c
$$

Substituting $c_{1}=v+c$ into Eq. (B1), the solution of Eq. (1) is

$$
w(x, t)=f(x-(c+v) t)
$$

We define

$$
F\left(x-v_{r} t\right)=f(x-(c+v) t)
$$

and so

$$
v_{r}=c+v
$$

which is the wave speed downstream in the direction of the string translation. Likewise, substituting $c_{1}=v-c$ into Eq. (B1), the solution of Eq. (1) is

$$
w(x, t)=f(x-(v-c) t)
$$

We define

$$
G\left(x+v_{l} t\right)=f(x-(v-c) t)=f(x+(c-v) t)
$$

and so

$$
v_{l}=c-v
$$


which is the wave speed upstream to the direction of the string translation.

So equation (1) has the general one-dimensional wave solution

$$
w(x, t)=F\left(x-v_{r} t\right)+G\left(x+v_{l} t\right)
$$

\section{References}

[1] K.F. Graff, Wave motion in elastic solids, Publication of Oxford University Press, London, 1975.

[2] W. A. Strauss, Partial differential equations: An introduction, Wiley, New York, 1992.

[3] T. Akkaya, W.T. van Horssen, Reflection and damping properties for semi-infinite string equations with non-classical boundary conditions, Journal of Sound and Vibration 336 (2015) 179-190.

[4] N.V. Gaiko, W.T. van Horssen, On wave reflections and energetics for a semi-infinite traveling string with a nonclassical boundary support, Journal of Sound and Vibration 370 (2016) 336-350.

[5] B. Yang, C.A. Tan, Transfer functions of one-dimensional distributed parameter systems. Journal of Applied Mechanics 59 (4) (1992) 1009-1014.

[6] C.A. Tan, S. Ying, Dynamic analysis of the axially moving string based on wave propagation. Journal of Applied Mechanics 64 (2) (1997) 394-400.

[7] W.T. van Horssen, On the influence of lateral vibrations of supports for an axially moving string. Journal of Sound and Vibration 268(2) (2003) 323-330.

[8] S.Y. Lee, M. Lee, A new wave technique for free vibration of a string with time-varying length. Journal of applied mechanics 69(1) (2002) 83-87.

[9] W.T. van Horssen, On the weakly damped vibrations of a string attached to a spring mass dashpot system. Journal of Vibration and Control 9(11) (2003) 1231-1248.

[10] Darmawijoyo, On the vibrations of a linear and a weakly 1-D wave equations with non-classical boundary damping. Journal of Applied Sciences 11(7) (2011) 1206-1212.

[11] E.W. Chen, N.S. Ferguson, Analysis of energy dissipation in an elastic moving string with a viscous damper at one end. Journal of Sound and Vibration 333(9) (2014) 2556-2570.

[12] N.V. Gaiko, W.T. van Horssen, On the transverse, low frequency vibrations of a traveling string with boundary damping. Journal of Vibration and Acoustics 137(4) (2015) 1316-1326.

[13] R.A. Sack, Transverse oscillations in travelling strings. British Journal of Applied Physics 5(6) (1954) 224-226.

[14] R.D. Swope, W. F. Ames, Vibrations of a moving thread line, Journal of the Franklin Institute 275 (1) (1963) 36-55.

[15] S.Y. Lee, C.D. Mote Jr. A generalized treatment of the energetics of translating continua, Part I: Strings and second order tensioned pipes. Journal of Sound and Vibration 204(5) (1997) 717-734. 